591-5.

9. Paterniti M.The most dangerous beauty. GQ 2002 Sep 28 [cited $2020 \mathrm{Apr}$ 18]. Available from: https://www.gq.com/story/pernkopfs-anatomynazi-history

10. Pernkopf E. ed, Ferner $\mathrm{H}$, transl Monsen $\mathrm{H}$. Atlas of topographical and applied human anatomy. Volume 1. Head and neck. 2nd revised ed. Baltimore-Munich:Urban \& Schwarzenberg; 1980.

11. Atlas M C: Ethics and access to teaching materials in the medical library: the case of the Pernkopf atlas. Bull Med Libr Assoc. 2001 Jan;89(1):51-8

12. Buklijas T. Culture of death and politics of corpse supply: anatomy in Vienna. 1848-1914 Bull Hist Med. 2008 Fall; 82(3):570-607

13. Pringle H. Anatomy. Confronting Anatomy's Nazi past. Science $2010 \mathrm{Jul}$ 16;329(5989):274-6.

14. Norton SA: On first looking into Pernkopf's atlas. (Part 1). Arch Dermatol. 2001 May:137(5):549-51

15. Norton SA: On first looking into Pernkopf's atlas. (Part 2). Arch Dermatol. 2001 Jul:137(7): 867-8.

16. Bagatur E. Nazi medicine - Part 2: the downfall of a profession and Pernkopf's anatomy atlas. Clin Orthop Relat Res. 2018 Nov; 476(11):21237.

17. Weindling P. Human guinea pigs and the ethics of experimentation: the $B M J$ 's correspondent at the Nuremberg medical trial. BMJ. 1996 Dec 7 313(7070):1467-70.

18. Riggs G. What should we do about Eduard Pernkopf's atlas? Acad Med. 1998 Apr; 73(4):380-6.

19. Israel HA: The Nazi origins of Eduard Pernkopf's Topographische Anatomie des Menschen: the biomedical ethical issues. Reference Librarian. 1998; 29(61/62):131-46. Epub
20. Vanderbrook AJ. Imperial Japan's human experiments before and during World War Two. University of Central Florida. Electronic Theses and Dissertations, 2004-2019. 2013[cited 2020 Aug 19].p 55. Available from: https://stars.library.ucf.edu/etd/2589

21. Moll FH, Krischel M. Albert Neisser and the first Prussian directive on informed consent. In:Sakti Das MD, Ed.:Skeletons in the closet:Indignities and injustices in medicine. Linthicum, Maryland:The American Urological Association; 2012.

22. Horner JS. Retreat from Nuremberg? Can we prevent unethical medical research? Public Health. 1999; 113:205-10.

23. Rodriguez MA. First, do no harm: The US sexually transmitted experiments in Guatemala. Am J Public Health. 2013:103:2122-6.

24. Appleyard B. Should we love the art if the artist is a monster? The Sunday Times. 2017 Dec 24[cited 2020 Aug 15]. Available from: https:// www.thetimes.co.uk/article/should-we-love-the-art-if-the-artist-is-amonster-nvrdmg3hd

25. Harding L. Von Hagens forced to return controversial corpses to China. The Guardian. 2004 Jan 23[cited 2020 Aug 16]. Available from: https:// www.theguardian.com/world/2004/jan/23/arts.china

26. Yee A, Hildebrandt S, Seidelman WE, Mackinnon SE. Letter to the editor: Nazi medicine - Part 2: The downfall of a profession and Pernkopf's anatomy atlas. Clin Orthop Relat Res. 2018; 476:2465-6.

27. Polak JA. Vienna protocol for when Jewish or possibly-Jewish human remains are discovered. In: Czech H, Drumi C, Weindling P, Eds. Medical ethics in the70 years after the Nuremberg Code, 1947 to the present. Cent Eur J Med. 2018 [cited 2020 Apr 14]; 130: S239-43. Available from: https://link.springer.com/content/pdf/10.1007/s00508-018-1343-y.pdf

\title{
Moving from clinical to pragmatic equipoise in health policy and systems research
}

\section{VIJAYAPRASAD GOPICHANDRAN}

\section{Abstract}

Health policy and systems research refers to the research conducted on the formulation, impact, organisation and functioning of health policies, and how to optimise the functioning of health systems and policies towards achieving health for all. There is emerging scholarship on the ethics of conducting such health policy and systems research. Ethics of health policy and systems research, though similar to the ethics of traditional clinical research in many ways, has several important distinctions. In traditional clinical research on human participants, where two treatments or interventions are compared, clinical equipoise is an important ethical consideration. This refers to

Author: Vijayaprasad Gopichandran (vijay.gopichandran@gmail.com), Assistant Professor, Department of Community Medicine, ESIC Medical College and PGIMSR, KK Nagar, Chennai 600078 INDIA.

To cite: Gopichandran V. Moving from clinical to pragmatic equipoise in health policy and systems research. Indian J Med Ethics. 2020 Oct-Dec;5(4) NS: 326-9. DOI: 10.20529/JMME.2020.77.

Published online on July 20, 2020.

Manuscript Editor: Sandhya Srinivasan, Veena Johari

( ) Indian Journal of Medical Ethics 2020 the genuine uncertainty among professional peers on whether one of the interventions is better than the other. This uncertainty is in the biomedical efficacy of the intervention. Unless such equipoise exists, clinical research is said to be unethical from the benefit-risk balance and justice perspectives. In health policy and systems research, the question of clinical equipoise is often not relevant. This article will describe the condition of clinical equipoise in health policy and systems research, its applications and challenges.

Key words: clinical equipoise, health policy and systems research, pragmatic equipoise

\section{Introduction}

Health policy and systems research (HPSR) refers to all research that attempts to understand the way health systems function and methods to strengthen them $(1,2)$. The important goals of HPSR are to understand the dynamics of functioning of a health system, to study how interventions impact the functioning and outputs of the system, to evaluate the influence of policies on health system functions and outcomes and to strengthen the health system through interventions and policies that are grounded in evidence. Therefore, HPSR is a multidisciplinary enterprise involving contributions from healthcare providers, public health experts, policy makers and 
people living in the community. HPSR involves research on health financing, governance mechanisms, service delivery, human resources for health, information technology, as well as the supply of drugs, devices and other utilities for optimal functioning of the health system (2). It also attempts to understand the norms, values and power dynamics within the health system that lead to its effective functioning. One of the unique characteristics of HPSR is that the research is situated in the real world context.

For the purpose of this article traditional clinical research will be defined as all research aimed at identifying the burden of disease, its distribution, severity, risk factors, clinical features, course of illness, treatment options, preventive interventions, long term outcomes, complications and prognosis. There are several important distinctions between traditional clinical research and HPSR. To explain with an example, a traditional clinical research study on the efficacy of a vaccine would conduct a vaccine trial in which healthy volunteers are allotted to two groups, one group receiving the new vaccine and the other receiving routine care. The difference in incidence of the infection will be studied to understand its efficacy. However, HPSR will look at issues such as logistics and supply chain of the vaccine to the community, how the acceptance of the vaccine in the community can be increased, what its pricing should be in the market, etc. Therefore, in the spectrum of health research, there is basic scientific research which begins in the laboratory, there is research on human participants which is carried out at the bedside in clinical trials, and finally there is HPSR which is research in the real world context.

There is emerging scholarship on the ethics of HPSR (1) Some of the key ethical considerations in HPSR, as in most forms of research on human participants, include upholding the autonomy of the individuals and communities which participate, establishing a fine balance between benefits and risks to individuals and communities, considerations of justice, responsiveness to the needs of the community and health system, sustainability, scalability and post-research commitment to the community and health system, and a strong community and stakeholder engagement from the stage of research design $(3,4)$.

One of the highly debated ethical issues in traditional clinical research has been the issue of clinical equipoise. In this commentary, I will attempt to frame the idea of clinical equipoise in the context of HPSR and discuss the challenges of using it as an ethical requirement to conduct HPSR research.

\section{The debate on clinical equipoise in traditional clinical research}

Clinical equipoise in the clinical trial context is a highly debated ethical issue and has evolved over the past 40 years from an absolute evidentiary basis for justifying treatments provided in the arms of a clinical trial (CT) to a relative notion that there exists a genuine professional disagreement among the community of experts on the best treatment. It is important to note that in clinical equipoise, the judgement regarding the relative merit of one treatment over the other is based purely on the biomedical efficacy of the intervention. In the early 1970's, Fried defined clinical equipoise as the clinician-researcher's belief that there is no evidence to support that either of the two (or more) interventions studied is superior to the other(s) (5). This is referred to as the absolute evidentiary criterion for clinical equipoise. However, such a stringent criterion of clinical equipoise makes the conduct of any $\mathrm{CT}$ extremely challenging because there always exists some evidence, sometimes from pre-clinical animal experiments, that one is better than the other. Moreover, even if equipoise does exist at the start of the $\mathrm{CT}$, theoretically the equipoise will increasingly be disturbed in the course of the trial, even in the absence of analysis. Freedman, in 1987, came up with a revision of the clinical equipoise criterion, where he proposed that rather than the individual clinician-researcher making the absolute evidentiary decision on which treatment is superior, the criterion should be a "genuine professional disagreement among a community of expert peers."(6)

The other point of debate in clinical equipoise is whose sense of equipoise matters? The clinician-researcher in a CT may feel there is justifiable clinical equipoise based on the biomedical knowledge that is available, but if the patient-participant in the research prefers one treatment to the other because of its less invasive nature or other forms of acceptability, then can that equipoise really be said to exist? This debate raises the issue of the patient's preferences contributing to decisions on whether equipoise exists or not. In the era of patient centred care where patients and communities must be equal partners in healthcare, health knowledge is understood to be embedded in communities. The judgement of patients and communities counts as support to evidence-based medicine and public health. This concept of equipoise further complicates the debate on clinical equipoise as a condition for ethically justified research. $(7,8)$

The main ethical principles that underpin the clinical equipoise condition are benefit-risk balance and justice. The presence of clinical equipoise ensures that benefits are optimised, risks are minimised and there is fairness in the distribution of benefits and risks between the arms in the CT. If equipoise does not exist, then one of the two arms in the $\mathrm{CT}$ will be intentionally subject to an inferior treatment compared to the other arm. This goes against the principle of risk-benefit balance and justice in the CT. However, the idea of clinical equipoise in HPSR requires a different framing.

\section{Challenges of clinical equipoise in HPSR}

I will use the example of a typical implementation research in the HPSR paradigm to illustrate how the presence of clinical equipoise is not necessary or useful. Subsequently I will also illustrate another HPSR in which clinical equipoise is necessary. With the help of these two examples, I will illustrate the challenges in using the traditional understanding of biomedical clinical equipoise to ethically justify HPSR and advocate for a reframing into a "pragmatic equipoise". 
Researchers wanted to study the implementation of the Home Management of Malaria (HMM) programme in Burkina Faso. HMM involves early identification and treatment of malaria, especially among children under 5 years of age at their own homes. Community Health Workers (CHWs) provide the care at the community level, thus making malaria treatment readily accessible to the community. Several research studies had already demonstrated the feasibility and effectiveness of this HMM strategy. (9-11) There is also evidence that in areas with rampant chloroquine resistance, artemisinin combination therapy could also be delivered using the HMM strategy. This study in Burkina Faso was intended to understand implementation issues in the local context. A total of fourteen community clinics were allotted, seven to the intervention arm, in which the CHWs were trained to provide HMM and seven to the control arm, in which there were no CHWs. The artemisinin combination therapy was made available at the clinic level in all the fourteen clinic centres. The study demonstrated that CHWs could successfully deliver the HMM and thus reduce the burden on the staff at the health facility level (12).

If we look closely at the intervention and control arms in this trial, clinical equipoise in its pure biomedical sense does not exist. The intervention arm has HMM provided by $\mathrm{CHWs}$. The control arm has no HMM, which is actually routine care in Burkina Faso. Previous studies have shown HMM to substantially improve access to anti-malarial drugs in communities. Therefore, there is no genuine disagreement on the effectiveness of HMM. However, the equipoise condition is not meaningful in this context because the main objective of the study is not to establish the biomedical efficacy of HMM, but to study the factors influencing its implementation, whether it reduces the load on the health facilities; whether it increases the treatment of malaria at community level; and whether the utilisation of HMM is good in Burkina Faso.

One could raise the question whether such a study in which one group is denied access to a superior intervention is required. In the HPSR context, this is a very important question. The control group in this case is receiving routine care, which is availability of anti-malarial treatment in the clinics. Therefore, they are not denied the standard care. The intervention group is receiving HMM, which is new, and its effectiveness will depend on the social context. It may or may not be acceptable to people. It may or may not work out well in terms of logistics. If it doesn't work out successfully, the control arm might turn out to be better than the intervention arm. Unless it is compared with routine care, even though it is inferior, it is difficult to understand whether the new intervention works, and if it works how much more effective it is than routine care. Such details are important to make decisions on scaling up of the intervention.

One could also argue that there indeed exists an uncertainty about whether the HMM intervention will work in Burkina Faso. However such an uncertainty is not the uncertainty about the biomedical efficacy of HMM, but rather the social, political, cultural and contextual uncertainty. This calls for a reframing of the concept of clinical equipoise in HPSR as "pragmatic equipoise" or contextual equipoise which is the genuine uncertainty as to whether the intervention will work in the real world context of Burkina Faso as it did in other countries.

In some situations, clinical equipoise may be both necessary and feasible in HPSR. I will take a more recent example of a study where the clinical equipoise condition was attempted in an HPSR cluster randomised controlled trial. A study was designed among 40 community health centres (CHCs) of the states of Haryana and Karnataka, where the $\mathrm{CHCs}$ were randomly assigned to the intervention, a mobile phone based health application (mHealth) system for electronic data capture, storage and mobile based decision support system for integrated management of hypertension, diabetes, tobacco use, alcohol use and depression; or the control which involved training of physicians for clinical management of these five conditions, display of treatment algorithms on the clinic walls, training of nurses and provision of a tablet PC for electronic data capture by the nurses.

The study did not find any incremental benefit of the mHealth intervention over enhanced routine care (13). In this study clinical equipoise can be said to exist because the two arms received interventions which were similar - capacity building of health care providers in delivering care for patients with non-communicable diseases, using two modalities. While there is no uncertainty that capacity building will have effect, there was genuine uncertainty about which of the two interventions would work out better. The clinical equipoise was feasible because both interventions involved similar content delivery albeit through different modalities. The two modalities were distinctly different and therefore there is a genuine uncertainty about which of the two would work better from a purely biomedical as well as socio-cultural perspective. One could argue that it is because of comparison with enhanced care for the control group that the mHealth intervention did not show incremental benefit. However, the goal of the study was to understand the most effective strategy for management of non-communicable diseases at a lower cost by providing capacity building of the physicians at primary care level; and therefore leaving the control group to receive only routine care could not have achieved this goal.

\section{Possible solutions to the challenges of clinical equipoise in HPSR}

One of the solutions to the problem of clinical equipoise for HPSR is to understand the concept differently considering the unique characteristics of the research goals. Often in HPSR the research goals are related to how best to adopt a new health policy, or how to optimise the health system to improve health outcomes. In such a context it is understood that basic efficacy, safety, effectiveness studies of the intervention are already completed. In other words, there is no reason to believe that the intervention is ineffective, and the criterion of uncertainty from the biomedical efficacy point of view is nonexistent. Therefore, HPSR must he held to a different standard of equipoise, ie pragmatic equipoise or contextual equipoise. 
Pragmatic equipoise is a practical (rather than theoretical) consideration. It asks "is there genuine uncertainty about the effectiveness of an intervention when implemented in the real world context?" This question should guide equipoise decisions in HPSR.

\section{Conclusion}

While conceptualising clinical equipoise in traditional clinical research, the goal was to ensure benefit-risk balance and justice as described above. However, the goals of HPSR are different and this warrants a careful reframing of the clinical equipoise condition. The original idea of clinical equipoise which was developed in the context of CT, if applied to HPSR is likely to lead to serious delays, high costs and unresponsiveness to the needs of the health systems and local communities. Many times, the clinical equipoise condition may not be feasible or necessary in HPSR as seen in the description above. Ethical guidance on HPSR must carefully consider the clinical equipoise criterion and must frame it in a manner that best suits HPSR in varied contexts.

\section{References}

1. Pratt B, Paul A, Hyder AA, Ali J. Ethics of health policy and systems research: A scoping review of the literature. Health Policy Plan. 2017 Jul 1;32(6):890-910.

2. Gilson L, Hanson K, Sheikh K, Agyepong IA, Ssengooba F, Bennett S. Building the field of health policy and systems research: social science matters. PLoS Med.2011 Aug;8(8):e1001079.

3. Luyckx VA, Biller-Andorno N, Saxena A, Tran NT. Health policy and systems research:towards a better understanding and review of ethical issues. BMJ Glob Health. 2017 Jun 10;2(2):e000314.

4. Gopichandran V, Luyckx VA, Biller-andorno N, Fairchild A, Singh J, Tran $\mathrm{N}$, et al. Developing the ethics of implementation research in health. Implement Sci.2016 Dec 9;11(1):161. DOI:10.1186/s13012-016-0527-y.

5. Fried C. Miller FG, Wertheimer A, editors. Medical experimentation: Personal integrity and social policy: New edition. Oxford University Press; 2016.

6. Freedman B. Equipoise and the ethics of clinical research. $N$ Engl J Med. 1987 Jul;317(3):3-16. DOI: 10.1056/NEJM198707163170304.

7. Miller FG, Brody H. Clinical equipoise and the incoherence of research ethics. J Med Philos. 2007 Mar-Apr;32(2):151-65.

8. Miller FG, Brody H. A critique of clinical equipoise: therapeutic misconception in the ethics of clinical trials. Hastings Cent Rep. 2003 May-Jun;33(3):19-28.

9. Kidane G, Morrow RH. Teaching mothers to provide home treatment of malaria in Tigray, Ethiopia: a randomised trial. Lancet. 2000 Aug 12;356(9229):550-5.

10. Pagnoni F, Convelbo N, Tiendrebeogo J, Cousens S, Esposito F. A community-based programme to provide prompt and adequate treatment of presumptive malaria in children. Trans $R$ Soc Trop Med Hyg.1997 Sep-Oct;91(5):512-7.

11. Sirima SB, Konate A, Tiono AB, Convelbo N, Cousens S, Pagnoni F. Early treatment of childhood fevers with pre packaged antimalarial drugs in the home reduces severe malaria morbidity in Burkina Faso. Trop Med Int Heal. 2003 Feb 1;8(2):133-9.

12. Tiono $A B$, Kaboré $Y$, Traoré $A$, Convelbo $N$, Pagnoni $F$, Sirima $S B$. Implementation of Home based management of malaria in children reduces the work load for peripheral health facilities in a rural district of Burkina Faso. Malar J. 2008 Oct 3;7(1):201.DOI:10.1186/1475-2875-7201.

13. Prabhakaran D, Jha D, Prieto-Merino D, Roy A, Singh K, Ajay VS, et al. Effectiveness of an mHealth-based electronic decision support system for integrated management of chronic conditions in primary care: the mWellcare cluster-randomized controlled trial. Circulation. 2019 Jan 5;139(3):380-91. 